\title{
Were ancestral proteins less specific?
}




\section{Abstract}

Some have hypothesized that ancestral proteins were, on average, less specific than their 9 descendants. If true, this would provide a universal axis along which to organize protein 10 evolution and suggests that reconstructed ancestral proteins may be uniquely powerful tools 11 for protein engineering. Ancestral sequence reconstruction studies are one line of evidence 12 used to support this hypothesis. Previously, we performed such a study, investigating the 13 evolution of peptide binding specificity for the paralogs S100A5 and S100A6. The modern 14 proteins appeared more specific than their last common ancestor (ancA5/A6), as each par- ${ }_{15}$ alog bound a subset of the peptides bound by ancA5/A6. In the current study, we revisit ${ }_{16}$ this transition, using quantitative phage display to measure the interactions of 19,194 ran- ${ }^{17}$ dom peptides with human S100A5, S100A6, and ancA5/A6. This unbiased screen reveals 18 a different picture. While S100A5 and S100A6 do indeed bind to a subset of the peptides 19 recognized by ancA5/A6, they also acquired new peptide partners outside of the set recog- 20 nized by ancA5/A6. Our previous work showed that ancA5/A6 had lower specificity than its 21 descendants when measured against biological targets; our new work shows that ancA5/A6 22 has similar specificity to the modern proteins when measured against a random set of peptide ${ }_{23}^{23}$ targets. This demonstrates that altered biological specificity does not necessarily indicate 24 altered intrinsic specificity, and sounds a cautionary note for using ancestral reconstruction 25 studies with biological targets as a means to infer global evolutionary trends in specificity. 26

\section{Introduction}

Changes in protein specificity are essential for evolution (Alhindi et al. , 2017; Carroll et al. , 28 2008; Clifton \& Jackson, 2016; Kaltenbach \& Tokuriki, 2014; Kanzaki et al. , 2012; Kherson- 29 sky \& Tawfik, 2010; Reinke et al. , 2013; Soskine \& Tawfik, 2010). One intriguing suggestion 30 is that, on average, proteins become more specific over evolutionary time (Copley, 2012; 31 Jensen, 1976; Khersonsky \& Tawfik, 2010; Wheeler et al. , 2016). If true, this would be 32 a directional "arrow" for protein evolution (Gaucher et al. , 2008; Mannige et al. , 2012; 33 Risso et al. , 2014; Wheeler et al. , 2016). Such proposed trends are controversial (Wheeler 34 
et al. , 2016; Williams et al. , 2006), but could ultimately provide fundamental insights into 35 the evolutionary process. For example, increasing specificity might indicate that proteins 36 become less evolvable over time, as they have fewer promiscuous interactions that can be 37 exploited to acquire new functions (Copley, 2012; Khersonsky \& Tawfik, 2010). From a prac- 38 tical standpoint, it has also been suggested that less-specific reconstructed ancestors would 39 be powerful starting points for engineering new protein functions (Risso et al. , 2013). 40

There are several reasons that proteins may, on average, evolve towards higher specificity. $\quad 41$ First, gene duplication followed by subfunctionalization could lead to a partitioning of ances- 42 tral binding partners between descendants, and thus increase specificity along each lineage 43 (Alhindi et al. , 2017; Clifton \& Jackson, 2016; Eick et al. , 2012; Hittinger \& Carroll, 2007). 44 Second, as metabolic pathways and interaction networks become more complex, proteins 45 must use more sophisticated rules to "parse" the environment: if an ancestral protein had to 46 discriminate between fewer targets than modern proteins, it could be less specific and still ${ }_{47}$ achieve the same biological activity (Eick et al. , 2012). Finally, on the deepest evolutionary 48 timescales, it has been pointed out that the proteome of the last universal common ancestor $\quad 49$ was small. As a result, each protein would have been required to perform multiple tasks and 50 hence have lower specificity (Copley, 2012; Jensen, 1976).

Much of the empirical support for the increasing-specificity hypothesis comes from an- 52 cestral reconstruction studies (Alhindi et al. , 2017; Carroll et al. , 2008; Clifton \& Jackson, 53 2016; Devamani et al. , 2016; Eick et al., 2012; Ma et al. , 2016; Pougach et al. , 2014; Rauw- ${ }^{54}$ erdink et al. , 2016; Risso et al. , 2013, 2014; Wheeler et al. , 2017; Zou et al. , 2015). The 55 results from one such study are shown schematically in Fig 1A. We previously studied the 56 evolution of peptide binding specificity in the amniote proteins S100A5 and S100A6. These ${ }_{57}$ proteins bind to $\approx 12$ amino acid linear peptide regions of target proteins to modulate their $\quad 58$ activity (Fig 1A) (Bertini et al. , 2009; Donato et al. , 2013; Leclerc et al. , 2009; Lee et al. , 59 2008; Liriano, 2012; Santamaria-Kisiel et al. , 2006; S \lomnicki et al. , 2009; Streicher et al. , 60 2009). These binding interactions tend to have $K_{D}$ in the $\mu M$ range. We found that S100A5 61 and S100A6 orthologs bound to distinct peptides, but that the last common ancestor bound 62 to all of the peptides we tested (Fig 1A) (Wheeler et al. , 2017). Other studies, probing ${ }_{63}$ other classes of interaction partners, have found similar results: the ancestor interacts with 64 
a broader range of partners than extant descendants (Alhindi et al. , 2017; Carroll et al. , 65 2008; Clifton \& Jackson, 2016; Devamani et al. , 2016; Eick et al. , 2012; Ma et al. , 2016; 66 Pougach et al. , 2014; Rauwerdink et al. , 2016; Risso et al. , 2013, 2014; Wheeler et al. , 67 2017; Zou et al. , 2015).

But do such experiments truly test the increasing-specificity hypothesis? The hypothesis $\quad 69$ can be represented as a Venn diagram: the set of targets recognized by the ancestor is larger 70 than the sets of targets recognized by its descendants (Fig 1B). Results such as those in Fig ${ }_{71}$ 1A are not, however, sufficient to resolve this Venn diagram. Fig 1C illustrates two radically 72 different Venn diagrams consistent with our experimental observations of peptide binding $\quad 73$ in Fig 1A. One possibility is increasing specificity (the descendant sets are smaller than the 74 ancestral set). Another possibility is shifting specificity (the descendant sets remain the 75 same size but diverge in their composition). Testing only a small or biased set of binding 76 partners could lead to incorrect conclusions about the evolutionary process. Distinguishing 77 the possibilities shown in Fig $1 \mathrm{C}$ requires estimating the populations in each region of the 78 Venn diagram, which can only be done with a much larger, unbiased sample of the set of 79 binding partners. 


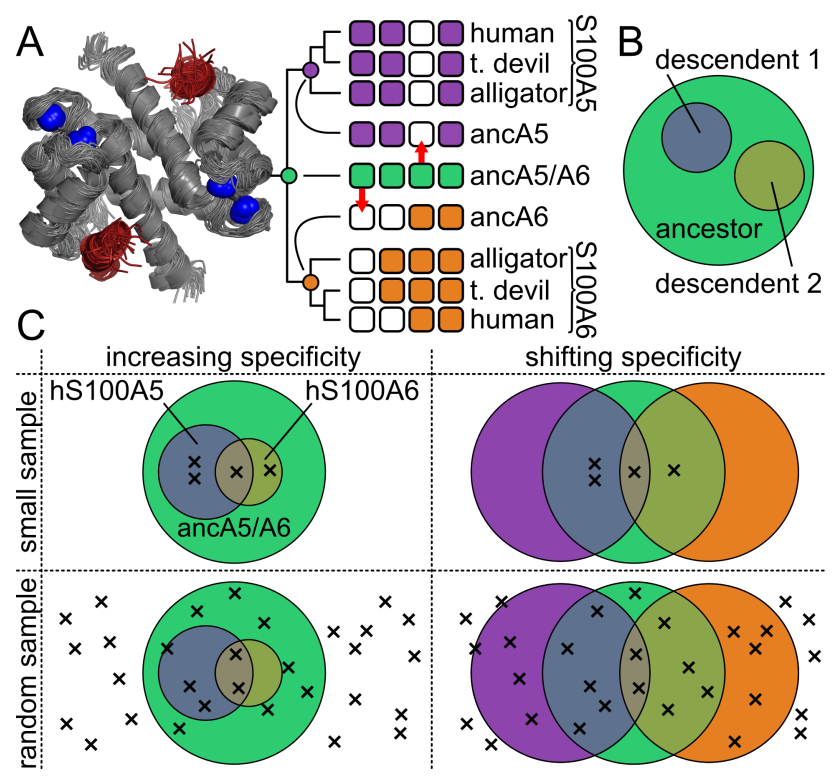

Fig 1. Testing the increased specificity hypothesis requires unbiased sampling 83 of targets. A) Experimentally measured changes in peptide binding specificity for S100A5 $\quad 84$ and S100A6 (taken from (Wheeler et al. , 2017)). Structure: location of peptide (red) 85 binding to a model of S100A5 (gray, PDB: 2KAY). Bound $\mathrm{Ca}^{2+}$ are shown as blue spheres. ${ }_{86}$ Phylogeny: Boxes represent binding of four different peptides (arranged left to right) to 87 nine different proteins (arranged top to bottom). A white box indicates the peptide does 88 not bind that protein; a colored box indicates the peptide binds. Colors denote ancA5/A6 ${ }_{89}$ (green), S100A5 (purple), and S100A6 (orange). Red arrows highlight ancestral peptides 90 lost in the modern proteins. B) Venn diagram of the increasing-specificity hypothesis. The 91 large circle is set of targets recognized by the ancestor; the smaller circles are sets of targets $9_{92}$ represented its descendants. C) Venn diagrams show overlap in peptide binding sets between ${ }_{93}$ ancA5/A6, S100A5, and S100A6. Crosses denote experimental observations. Columns show 94 two evolutionary scenarios: increasing specificity (left) versus shifting specificity (right). 95 Rows show to different sampling methods: small sample (top) versus random sampling 96 (bottom). Colors are as in panel B. (1) . .

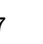
sis

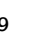
. in (2)

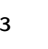

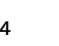

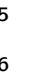


To test for the evolution of increased specificity, we set out to estimate changes in the 98 total set of peptides between ancA5/A6 and two of its descendants - human S100A5 (hA5) 99 and human S100A6 (hA6). This evolutionary transition is an ideal model to probe this 100 question. We already have a reconstructed ancestral protein that exhibits an apparent gain 101 in specificity over time for both proteins, at least for a small collection of peptides (Wheeler 102 et al. , 2017). Further, because they bind to $\approx 12$ amino acid peptides, the set of binders is 103 discrete and enumerable $\left(20^{12}=4 \times 10^{15}\right.$ targets $)$. This contrasts with interactions between 104 proteins and small molecules, for which there is an effectively infinite chemical "space" of 105 possible moieties to sample. We therefore set out to estimate changes in the total sets of 106 partners recognized by these proteins using a high-throughput characterization of peptide 107 binding. We found that the modern proteins bound to a similar number of targets as 108 the ancestor, and that both hA5 and hA6 acquired a large number of new targets since 109 ancA5/A6. Thus, the original observation that a smaller number of targets bound by the 110 ancestor relative to the modern proteins reflects a shifting set of targets - not a shrinking 111 set. This suggests that the evidence for a global trend towards increased specificity from 112 less-specific ancestral states should be revisited.

\section{Results}

\section{Peptide/protein interactions measured by phage display}

Our goal was to measure changes in the total binding sets between human S100A5 (hA5), hu- ${ }_{116}$ man S100A6 (hA6), and their last common ancestor (ancA5/A6) (Wheeler et al. , 2017). To 117 account for uncertainty in the reconstruction, we also characterized an alternate reconstruc- 118 tion of ancA5/A6 (altAll) that incorporates alternate amino acids at uncertain positions in 119 the reconstruction (Eick et al. , 2017). This protein differs at 21 of 86 sites from ancA5/A6, 120 but behaved similary to ancA5/A6 in our previous expereiments (Wheeler et al. , 2017). 121

We first assayed the interaction of tens of thousands of peptides to each protein using 122 phage display. We panned a commercial library of randomized 12-mer peptides expressed as ${ }_{123}$ fusions with the M13 phage coat protein. The S100 peptide-binding interface is only exposed 124 
upon $\mathrm{Ca}^{2+}$-binding (Fig 1A); therefore, we performed phage panning experiments in the 125 presence of $\mathrm{Ca}^{2+}$ and then eluted the bound phage using EDTA (Fig 2A). The population 126 of enriched phage will be a mixture of phage that bind at the site of interest and phage that $\quad 127$ bind adventitiously (blue and purple phage, Fig 2A). Peptides in this latter category enrich 128 in $\mathrm{Ca}^{2+}$-dependent manner through avidity or binding at an alternate site (Sidhu et al. , 129 2000; Willats, 2002). To separate these populations, we repeated the panning experiment 130 in the presence of a saturating concentration of competitor peptide known to bind at the ${ }_{131}$ site of interest (Fig 2B) (Wheeler et al. , 2017). This should lower enrichment of peptides 132 that bind at the site of interest, while allowing any adventitious interactions to remain. By 133 comparing the competitor and non-competitor pools, we can distinguish between actual and 134 adventitious binders. 


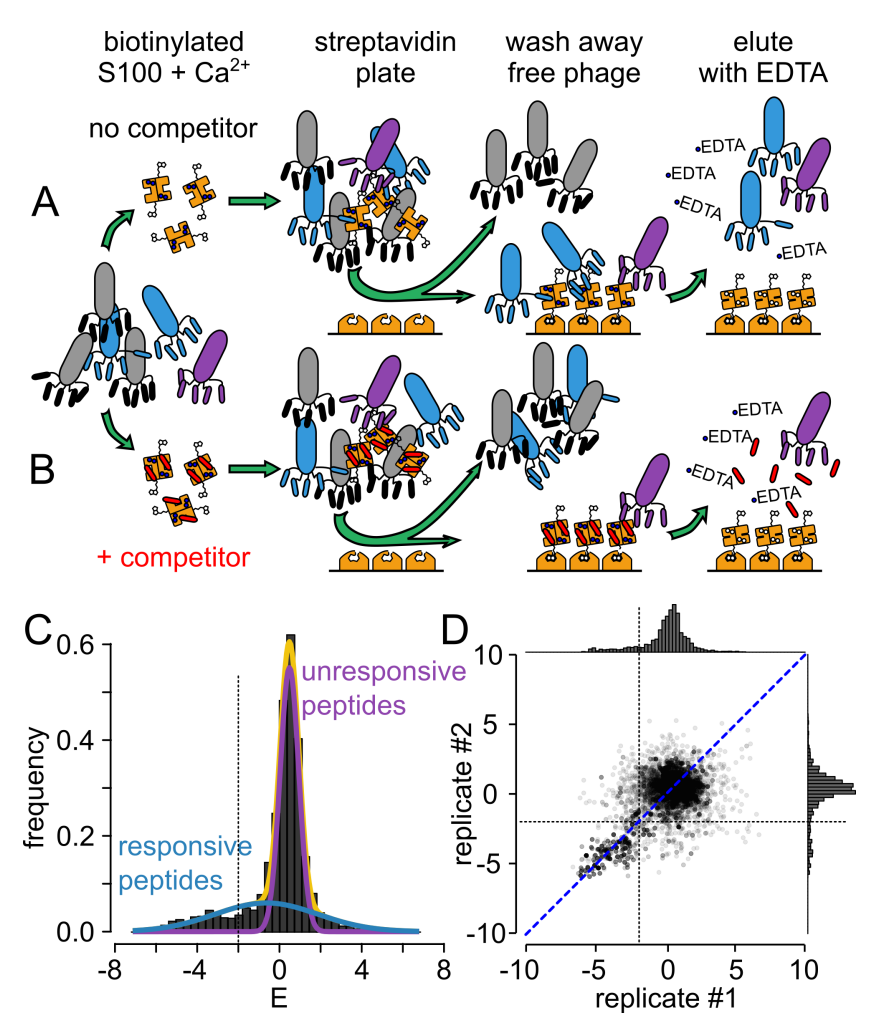

Fig 2. Set of binding peptides can be estimated using phage display. Rows show two different experiments, done in parallel, for each protein. Biotinylated, $C a^{2+}$-loaded, S100 is added to a population of phage either alone (row A) or with saturating competitor peptide added in trans (row B). Phage that bind to the protein (blue or purple) are pulled down using a streptavidin plate. Bound phage are then eluted using EDTA, which disrupts the peptide binding interface. In the absence of competitor (row A), phage bind adventitiously (purple) as well as at the interface of interest (blue). In the presence of competitor (row B), only adventitious binders are present. C) Distribution of enrichment values for peptides taken from pooled biological replicates of hA5. The measured distribution (gray) can be ${ }_{145}$ fit by the sum of two Gaussian distributions: responsive (blue) and unresponsive (purple), 146 which sum to the total (yellow). The dashed line indicates cutoff for $E$ values above which ${ }_{147}$ the probability the value arose from the unresponsive distribution is $<0.05$. D) Enrichment ${ }_{148}$ values from biological replicates are strongly correlated. Axes are enrichment for replicate \#1 149 or replicate $\# 2$. Points are individual peptides. Distributions for each replicate are shown on 150 the top and right, respectively. The blue dashed line is the best fit line (orthoganol distance 151 regression), explaining $81 \%$ of the variation in the data. (1)

.
西 . 152 
We performed this experiment with and without competitor, in biological duplicate, for ${ }_{153}$ all proteins. We found that phage enriched strongly for all proteins relative to a biotin-only 154 control (Fig S1). Further, the addition of competitor binding knocked down enrichment in 155 all samples (Fig S1). After panning, we sequenced the resulting phage pools, as well as the 156 input library, using Illumina sequencing. We applied strict quality control, discarding any 157 peptide that exhibited less than six counts (see methods, Fig S2). After quality control, we 158 had a total of 265 million reads spread over 17 samples (Table S1).

We estimated changes in the frequencies of peptides between samples with and without 160 competitor peptide. For each peptide $i$, we determined $E_{i}=-\ln \left(\beta_{i} / \alpha_{i}\right)$, where $\beta_{i}$ and $\alpha_{i}$ are ${ }_{161}$ the frequencies of the peptide in the non-competitor and competitor samples, respectively. 162 Defined this way, a more negative value of $E$ corresponds to a larger decrease in peptide ${ }_{163}$ frequency upon addition of a saturating amount of competitor peptide. More directly: the 164 more negative $E$ is for a peptide/protein pair, the better the protein is at selectively enriching 165 the peptide through an interaction at the canonical S100 peptide binding interface. We 166 estimated $E$ for $\approx 40,000$ different peptides for each protein (see methods, Fig S3). $\quad{ }_{167}$

We found that the distribution of $E$ for each protein could be described using two Gaus- ${ }_{168}$ sian distributions, apparently reflecting two underlying processes (Fig 2C, Fig S4). The 169 dominant peak, centered about $E=0$, consists of "unresponsive" peptides whose frequencies 170 change little in response to competitor peptide. A second, broader, distribution describes 171 "responsive" peptides whose frequencies change with the addition of competitor. For all pro- 172 teins, the responsive distribution was shifted towards negative values, meaning the addition 173 of competitor tends to knock off previously bound peptides. Using the mean and standard ${ }_{174}$ deviations of the responsive and unresponsive distributions, we could estimate the posterior 175 probability that a peptide with a given value of $E$ arose from the responsive rather than 176 unresponsive distribution, and thus reflects specific enrichment of a peptide. We found that 177 $p=0.05$ occurred around $E=-1.5$ for all proteins (Fig 2C, Fig S4, Fig S5). We therefore 178 interpret peptides exhibiting $E \leq-1.5$ interacting with the protein at a site that is dis- 179 rupted by addition of competitor peptide. Values of $E>-1.5$ are exhibited by unreponsive 180 peptides were thus not considered further.

There was no systematic difference between estimates of $E$ between biological replicates. 182 
We used orthogonal distance regression to compare values of $E$ for peptides seen in both 183 biological replicates. The slopes of these lines ranged from 0.9 to 1.1, with intercepts between 184 -0.09 and 0.11. hA5, for example, had a slope 1.06 and an intercept of -0.05 (Fig 2D). 185 There are two distinct regions in these correlation plots, corresponding to the unresponsive 186 and responsive peptide distributions. The unresponsive distribution forms a large cloud ${ }_{187}$ about zero. In contrast, the responsive peptide distribution extends along the 1:1 line in a 188 correlated fashion. If we focus on values of $E<-1.5$ - peptides arising from the "responsive" 189 distribution - the $1: 1$ axis of variation explains $87.8 \%$ of the total variation in the data 190 for hA5. The worst correlation, observed for altAll, was 75.8\%. Finally, because we are 191 comparing changes in the interaction sets between hA5, hA6 and the ancestral proteins, we 192 identified the set of 19,194 peptides that were observed for all four proteins and used this 193 set for our downstream analyses.

\section{Different changes in specificity occurred along the hA5 and hA6 lin- ${ }_{195}$} eages

We were now in a position to determine how specificity changed over time for these proteins. 197 We first measured the total size of the sets of peptides bound to each protein. If the modern 198 proteins are more specific than their ancestral protein, we would predict they would interact 199 with smaller sets of peptides than the ancestor.

Fig 3A shows the cumulative number of peptides with the $E$ below the indicated cutoff. 201 We found that ancA5/A6 interacts with $\approx 1,400$ peptides with $E \leq-1.5$ (green curve, 202 Fig 3A). Its highest enriching peptide has a score near $E=-5.5$. hA6 has a smaller set ${ }_{203}$ of peptides than ancA5/A6 for most values of $E$ (orange curve, Fig 3A), in line with the 204 increased specificity prediction. For example, ancA5/A6 has $\approx 500$ peptides with $E \leq-3,205$ while hA6 has only $\approx 300$ peptides over the same interval. hA5, in contrast, has a larger 206 interaction set for all values of $E$ (purple curve, Fig 3A). Its highest enriching peptide has 207 $E=-6.5$ and it has $\approx 1,700$ peptides with $E \leq-1.5$. This indicates that hA5 is not 208 binding a simple subset of the peptides bound by the ancestor. 209 
A

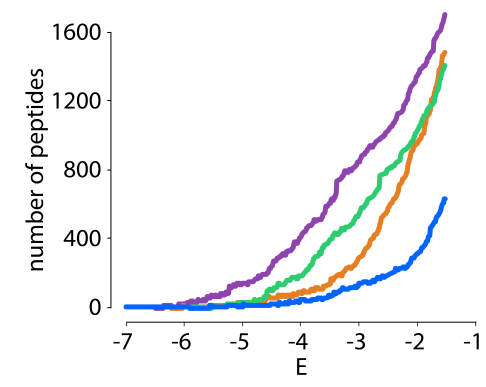

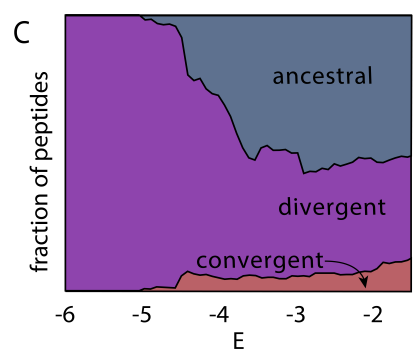

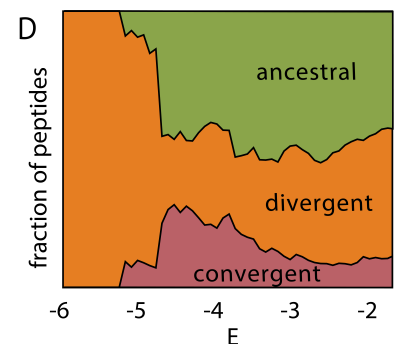

B ancA5/A6
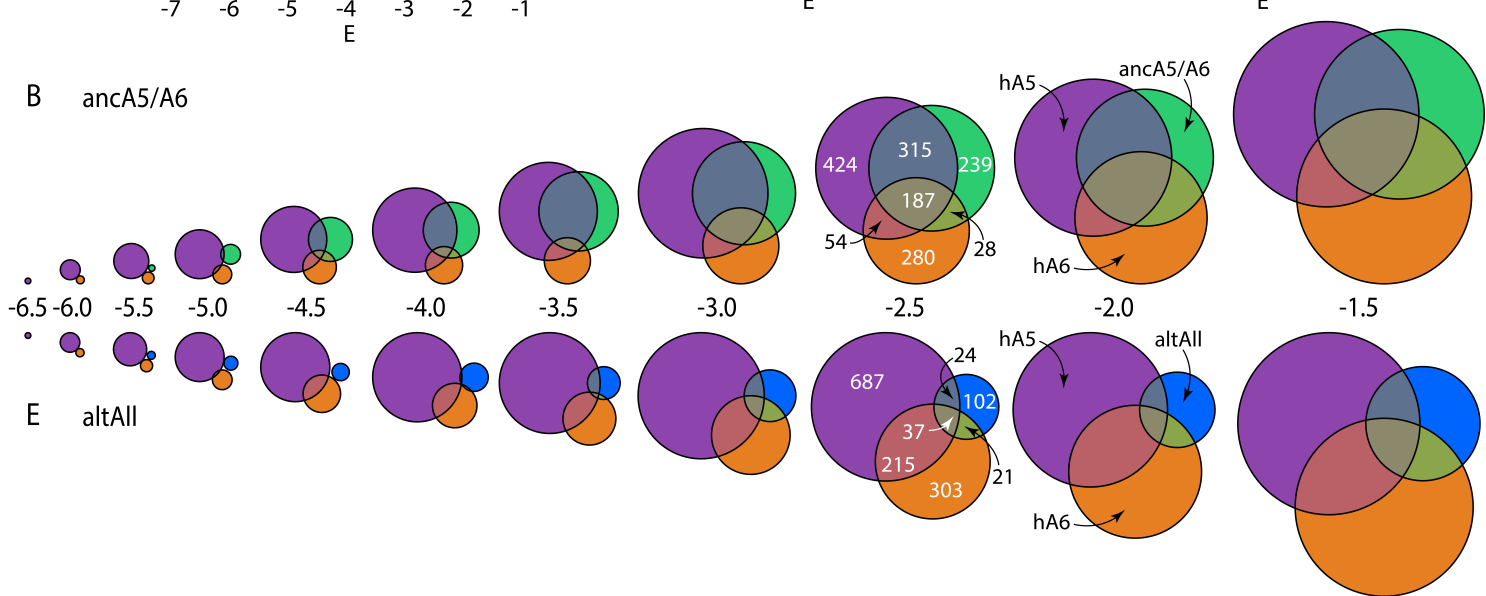

Fig 3. hA5 and hA6 gained new targets since their last common ancestor. A) 212 Cumulative number of peptides observed at or below each enrichment level for hA5 (purple), 213 hA6 (orange), ancA5/A6 (green), and altAll (blue). B) Venn diagrams for overlap between 214 cumulative number peptides observed at or below the indicated enrichment cutoff for hA5 215 (purple), hA6 (orange) and ancA5A6 (green). The areas are proportional to the number ${ }_{216}$ of peptides. The absolute numbers are shown for the $E \leq-2.5$ Venn diagram for scale. 217 C) Fraction of hA5 targets that overlap with ancA5/A6 ("ancestral"; slate), overlap with ${ }_{218}$ neither ancA5/A6 nor hA6 ("divergent"; purple), or overlap with hA6 but not the ancestor 219 ("convergent"; salmon). D) Fraction of hA6 targets that overlap with ancA5/A6 ("ancestral"; 220 green), overlap with neither ancA5/A6 nor hA5 ("divergent"; orange), or overlap with hA5 221 but not the ancestor ("convergent"; salmon). E) Venn diagrams for overlap between cumula- 222 tive number peptides observed at or below the indicated enrichment cutoff for hA5 (purple), 223 hA6 (orange) and the altAll ancestor (blue). 
To determine if this result was robust to uncertainty in the construction, we also investi- ${ }_{225}$ gated the set of peptides interacting with the alternate version of the reconstructed ancestor, 226 altAll. This protein had a much smaller set of partners than the other three proteins we ${ }_{227}$ studied: it only interacts with $\approx 650$ peptides with $E \leq-1.5$. Thus, the result of hA5 ${ }_{228}$ gaining targets since the ancestor is robust to phylognetic uncertainty; the result of hA6 ${ }_{229}$ becoming more specific is not. If the altAll reconstruction is a better reflection of the evolu- ${ }^{230}$ tionary history of these proteins than ancA5/A6, both hA5 and hA6 appear to have gained ${ }_{231}$ new targets over time. 232

We next probed the overlap between ancA5/A6 and its descendants as a function of $E_{233}$ (Fig 3B). For the highest enriching peptides - the most negative values of $E$ - each protein ${ }^{234}$ has a unique set of peptides. At a cutoff of $E \leq-5.3$, for example, the hA5 set has $76{ }_{235}$ peptides, the hA6 set has 13 peptides, and the ancA5/A6 set has 15 peptides. None of these ${ }^{236}$ peptides overlap between the three protein sets. ${ }^{237}$

At lower stringency $E$, we observed overlap between the peptide sets for the three pro- ${ }^{238}$ teins. By $E \leq-2.5$, for example, hA5 interacts with 980 peptides. Of these, 424 are unique ${ }_{239}$ to hA5 and 502 are shared with ancA5/A6. To determine if this overlap could arise by 240 randomly sampling potential peptide binding partners, we simulated sampling 980 peptides 241 (the size of the hA5 set at this $E$ cutoff) and 769 peptides (the size of ancA5/A6 set at ${ }_{242}$ this $E$ cutoff) from 19,194 unique peptides (the number of peptides in the whole dataset). ${ }^{243}$ In these simulations, we observed an average of $39.6 \pm 6$ shared peptides. The the z-score ${ }^{244}$ for observing 502 overlapping peptides is 79.2 - giving strong evidence that the overlap is 245 non-random. We did all pairwise comparisons between the sets of hA5, hA6 and ancA5/A6 246 the Venn diagrams shown in Fig 3B. The worst $p$ we found was $2.9 \times 10^{-7}$ : the overlapping ${ }^{247}$ sets are thus highly significant. 248

This analysis revealed that hA5, hA6 and ancA5/A6 bind to small, but overlapping, 249 sets of all possible binding peptides (Fig 3B). We next set out to understand the nature of ${ }_{250}$ the evolution of these sets. How did hA5 and hA6 change since their last shared common ${ }^{251}$ ancestor? A visual inspection of the Venn diagrams reveals that hA5 and hA6 exhibit ${ }^{252}$ similar patterns: both have retained many interactions with the ancestral binding set, while ${ }_{253}$ also acquring new partners over time. To make better sense of this pattern, we split the ${ }^{254}$ 
interaction targets of hA5 and hA6 into three categories: ancestral (peptides shared with 255 ancA5/A6), convergent (peptides shared between hA5 and hA6, but not the ancestor), and 256 divergent (peptides that are not shared by any others). We then plotted this for hA5 and ${ }_{257}$ hA6 as a function of $E$ cutoff (Fig 3C,D). 258

We found that hA5 gave a clear pattern of divergent evolution (Fig 3C). If we look at ${ }_{259}$ moderately enriching peptides $(-4.0 \leq E \leq-1.5)$ we find that $\approx 50 \%$ of hA5's peptides are ${ }_{260}$ ancestral, $\approx 45 \%$ are divergent, and $\approx 5 \%$ are convergent. For the highest enriching peptides 261 $(E<-4.0)$, we see the fraction of divergent peptides climbs even higher; for $E \leq-5.3,{ }^{262}$ all hA5 peptides were unique and thus - apparently - divergent. One explanation for this 263 observation is a lack of sufficient sampling: maybe the overall fraction of divergent peptides ${ }^{264}$ is constant across $E$ values, but that the low numbers of peptides with low values of $E$ led to 265 a chance over-representation of divergent peptides. To probe for this possibility, we assumed 266 that hA5 had populations like those reflected for moderate enrichment (ancestral: 50\%, 267 divgerent: $45 \%$ and convergent: 5\%). We then walked down $E$ and sampled the appropriate 268 numbers of peptides for each E. (For example, we sampled a total of 394 hA5 peptides 269 for an $E$ cutoff of -4 and only 62 hA5 peptides for an $E$ cutoff of -5.5$)$. This allowed us 270 to calculated expected variation in the numbers of peptides in the ancestral, divergent, or ${ }_{271}$ convergent categories due to sampling. We found that the observed increase in the relative ${ }_{272}$ number of divergent peptides could not be explained by sampling for hA5 $\left(p=5.6 \times 10^{-16}{ }_{273}\right.$ for $E \geq-5.5$; Fig $4 \mathrm{~A}$ ). This indicates that hA5 has acquired an increased number of highly ${ }_{274}$ enriching peptides relative to its ancestral state. 275 

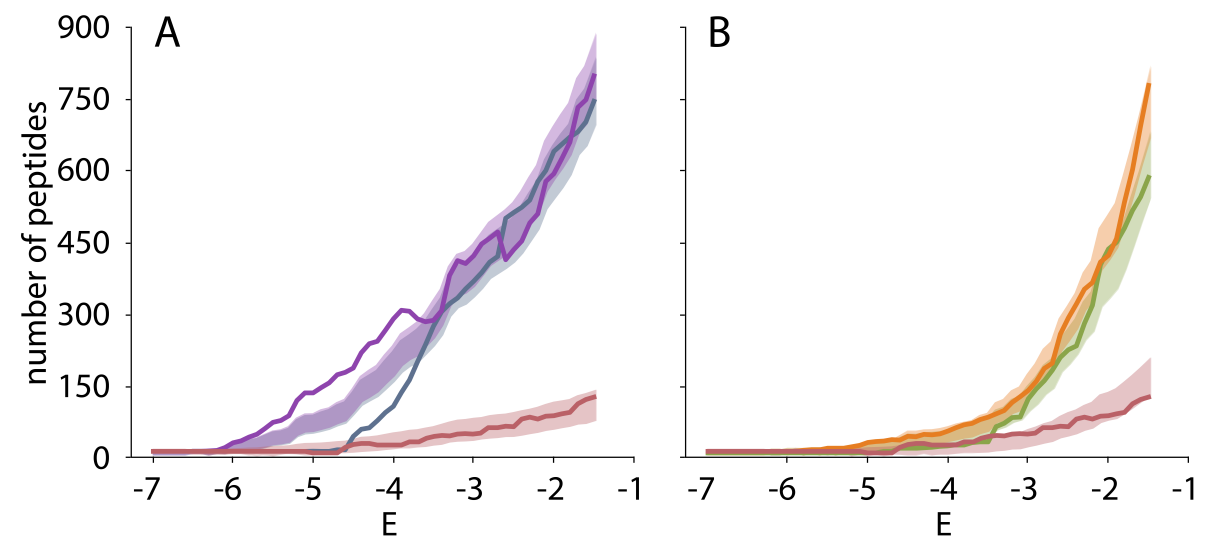

Fig 4. hA5 gained more highly enriched peptides since ancA5/A6. A) Cumulative 277 number of hA5 peptides observed at or below each enrichment level that are ancestral (slate), 278 divergent (purple), or convergent (salmon). The solid lines were observed experimentally 279 (seen in Fig 3C). The shaded regions indicate the 95\% confidence intervals for the number of ${ }_{280}$ expected peptides if the underlying proportions were ancestral (0.5), divergent (0.45), and 281 convergent (0.05). For $E<-4.0$, the observed number of divergent peptides is elevated ${ }_{282}$ above and the observed number of ancestral peptides is depressed below the expectation. B) ${ }_{283}$ Equivalent plot for hA6. Curves are ancestral (green), divergent (orange), and convergent 284 (salmon). There is no evidence for elevated numbers of divergent, highly enriched peptides 285 for hA6. 
We next turned our attention to hA6. Similar to hA6, it gave a pattern of divergent 287 evolution for moderately enriching peptides $(\approx 50 \%$ ancestral, $\approx 40 \%$ divergent, $\approx 10 \% \quad 288$ convergent) and then climbed to apparently 100\% divergent for the most negative values of 289 $E$ (Fig 3D). To see if this could be explained by the small numbers of peptides observed at 290 these values of $E$, we repeated the sampling analysis we performed for hA5 for hA6. For 291 hA6, saw no evidence that the relative proportions of divergent, convergent, and ancestral 292 categories changed as a function of enrichment (Fig 4B). Thus, we cannot resolve whether ${ }_{293}$ hA6 has acquired a relatively higher proportion of highly enriching targets since ancA5/A6. 294

Finally, to test the robustness of our conclusions to uncertainty in the reconstruction, we 295 repeated our analysis using the altAll ancestor (Fig 3E). We observed a similar pattern for 296 altAll and ancA5/A6. AltAll interacted with a small set of peptides that partially overlapped 297 with the hA5 and hA6 sets (Fig 3E). AltAll had a much smaller overlap with hA5 and hA6 298 than ancA5/A6. This small set only exacerbates the trends observed for ancA5/A6. For 299 example, over the interval from $-4.0 \leq E \leq-1.5$, an even greater fraction of hA5 peptides 300 are predicted to be divergent (40\% for ancA5/A6 vs. 90\% for altAll). Likewise, hA6 exhibits 301 a higher proportion of divergent peptides for altAll vs. hA6 (Fig 3E). 302

Thus, on both lineages, and with two different versions of the reconstructed ancestor, 303 we see that hA5 and hA6 did not gain specificity relative to their ancestral protein. Both 304 lineages maintained interactions with a large number of ancestral protein targets, but also 305 gained a set of new targets. Many of the newly acquired targets were specific to hA5 or 306 hA6, respectively, suggesting a pattern of divergent evolution. The set of partners shifted 307 and grew (hA5) or shifted and slightly shrank (hA6); neither lineage gave a pattern if simple 308 increasing specificity over time. 309

\section{Discussion}

In this work, we combined ancestral sequence reconstruction with a high-throughput assay 311 to measure evolutionary changes in the specificity of the proteins S100A5 and S100A6. In 312 a previous study of the biological binding partners of the modern proteins, we found that 313 specificity increased since the last common ancestor of the proteins (Fig 1). In the current 314 
study, we found the opposite: S100A5, in particular, became less specific over the same 315 evolutionary interval (Fig 3).

These results can be rationalized if we make our definition of specificity more precise. 317 Specificity may be viewed at two levels: biological and instrinsic. Biological specificity 318 measures the ability of a protein to parse its biological environment. It is determined by 319 both the affinity of a protein for its potential targets and the biological concentrations of 320 the protein and its targets. Such specificity can be tuned by selection, as altering biological 321 interactions can have a profound effect on an organism's fitness. 322

Intrinsic specificity, in contrast, measures the affinity of the protein for all possible targets, 323 regardless of whether a given target is encountered by the protein in a biological context. 324 As a whole, intrinsic specificity is invisible to selection: a mutation that alters the ability of 325 a protein to interact with a partner it never encounters will not affect fitness. These latent, 326 promiscuous, interactions can, however, set up future evolutionary change because the pre- 327 existing binding interaction can be exploited for new biological functionality (Copley, 2012; 328 Khersonsky \& Tawfik, 2010).

A protein with low intrinsic specificity may have a higher latent capacity to form new 330 interactions, potentially making it more "evolvable." If proteins indeed tend to gain instrinsic 331 specificity over time, one could even argue that they tend to become less evolvable. This 332 would be a striking evolutionary trend (Risso et al. , 2013, 2014). Practically, it would also 333 be a strong argument for using reconstructed ancestral proteins as the starting point for 334 protein engineering: a protein with lower instrinsic specificity would have a greater number 335 of latent interactions to exploit and optimize (Khersonsky \& Tawfik, 2010; Risso et al. , 336 2018).

Ancestral sequence reconstruction studies have, however, generally probed changes in 338 biological rather than instrinsic specificity (Alhindi et al. , 2017; Carroll et al. , 2008; Clifton 339 \& Jackson, 2016; Devamani et al. , 2016; Eick et al. , 2012; Ma et al. , 2016; Pougach et al. 340 , 2014; Rauwerdink et al. , 2016; Risso et al. , 2013, 2014; Wheeler et al. , 2017; Zou et al. 341 , 2015). Take our previous study on the evolution of S100A5 and S100A6 (Wheeler et al. , 342 2017). We selected known partners of S100A5 and S100A6, and then asked whether those 343 partners interacted with ancA5/A6. We found that ancA5/A6 bound all of the modern 344 
parterns, indicating that S100A5 and S100A6 did, indeed, acquire new biological specificity 345 relative to their ancestor (Fig 1$)$.

The current study, however, reveals that increased biological specificity need not imply 347 an increase in specificity. We found that human S100A5 and S100A6 both acquired many 348 new binding partners over the same interval they acquired new biological specificity. Hu- 349 man S100A5, in particular, binds to more targets than its ancestor: it's intrinsic specificity 350 decreased (Fig 3). This difference between our results for biological and intrinsic specifcity 351 suggests we must carefully define which form of specificity is under discussion when thinking 352 about global evolutionary trends.

Do we expect a trend in global specificity? In our view, the answer is "no." Because 354 intrinsic specificity is not under selection, the instrinsic specificity of the protein will be 355 determined by chance as the protein meanders through sequence space. Presumably, many 356 more sequences encode low intrinsic specificity than high instrinsic specificity proteins, just 357 as many more sequences encode low stability rather than high stability proteins (Taverna \& 358 Goldstein, 2002). As a result, we would expect most evolutionary steps to decrease, rather 359 than increase intrinsic specificity. 360

Given this, intrinsic specificity would only increase if it was somehow linked to some other 361 feature under selection. One might imagine, for example, that increasing biological specificity 362 necessarily increases intrinsic specificity for certain classes of ligands. It is, however, not 363 obvious that this will generally hold true. A mutation that changes biological specificity 364 alters the chemistry of a protein's binding interface, excluding some intrinsic partners and 365 adding others. We see no reason to assume the number of partners excluded would be 366 systematically higher than the number added for most classes of mutations and binding 367 sites.

Our work does not rule out a trend of increased intrinsic specificity over deep evolutionary 369 time, but it does caution against interpretating changes in biological specificity as evidence 370 for an overall trend. Testing for a global trend in intrinsic specificity will require studies of 371 unbiased sets of possible interaction partners. It will also necessitates studies of multiple 372 protein families, over deeper evolutionary time scales, and with different classes of binding 373 partners and substrates. 


\section{Materials and Methods}

\section{Molecular cloning, expression and purification in of S100 proteins}

Proteins were expressed in a pET28/30 vector containing an N-terminal His tag with a TEV 377 protease cleavage site (Millipore). For each protein, expression was carried out in Rosetta 378 E.coli (DE3) pLysS cells. 1.5 L cultures were inoculated at a 1:100 ratio with saturated 379 overnight culture. E.coli were grown to high log-phase $\left(O D_{600} \approx 0.8-1.0\right)$ with $250 \mathrm{rpm} 380$ shaking at $37^{\circ} \mathrm{C}$. Cultures were induced by addition of $1 \mathrm{mM}$ IPTG along with $0.2 \%$ glucose 381 overnight at $16^{\circ} \mathrm{C}$. Cultures were centrifuged and the cell pellets were frozen at $20^{\circ} \mathrm{C}$ and 382 stored for up to 2 months. Lysis of the cells was carried out via sonication on ice in $25 \mathrm{mM} 383$ Tris, $100 \mathrm{mM} \mathrm{NaCl}, 25 \mathrm{mM}$ imidazole, $\mathrm{pH}$ 7.4. The initial purification step was performed 384 at $4{ }^{\circ} \mathrm{C}$ using a $5 \mathrm{~mL}$ HiTrap Ni-affinity column (GE Health Science) on an Äkta PrimePlus 385 FPLC (GE Health Science). Proteins were eluted using a $25 \mathrm{~mL}$ gradient from 25-500 mM 386 imidazole in a background buffer of $25 \mathrm{mM}$ Tris, $100 \mathrm{mM} \mathrm{NaCl}, \mathrm{pH} 7.4$. Peak fractions were 387 pooled and incubated overnight at $4{ }^{\circ} \mathrm{C}$ with $\approx 1: 5 \mathrm{TEV}$ protease (produced in the lab). ${ }^{388}$ TEV protease removes the N-terminal His-tag from the protein and leaves a small Ser-Asn 389 sequence N-terminal to the wildtype starting methionine. Next hydrophobic interaction 390 chromatography (HIC) was used to purify the S100s from remaining bacterial proteins and 391 the added TEV protease. Proteins were passed over a $5 \mathrm{~mL}$ HiTrap phenyl-sepharose column 392 (GE Health Science). Due to the $\mathrm{Ca}^{2+}$-dependent exposure of a hydrophobic binding, the ${ }_{393}$ S100 proteins proteins adhere to the column only in the presence of $\mathrm{Ca}^{2+}$. Proteins were ${ }_{394}$ pre-saturated with $2 \mathrm{mM} \mathrm{Ca^{2+ }}$ before loading on the column and eluted with a $30 \mathrm{~mL}$ gradient 395 from $0 \mathrm{mM}$ to $5 \mathrm{mM}$ EDTA in $25 \mathrm{mM}$ Tris, $100 \mathrm{mM} \mathrm{NaCl}, \mathrm{pH} 7.4$.

Peak fractions were pooled and dialyzed against $4 \mathrm{~L}$ of $25 \mathrm{mM}$ Tris, $100 \mathrm{mM} \mathrm{NaCl}, \mathrm{pH} 397$ 7.4 buffer overnight at $4{ }^{\circ} \mathrm{C}$ to remove excess EDTA. The proteins were then passed once 398 more over the $5 \mathrm{~mL}$ HiTrap Ni-affinity column (GE Health Science) to remove any uncleaved 399 His-tagged protein. The cleaved protein was collected in the flow-through. Finally, protein 400 purity was examined by SDS-PAGE. If any trace contaminants appeared to be present we 401 performed anion chromatography with a $5 \mathrm{~mL}$ HiTrap DEAE column (GE). Proteins were ${ }_{402}$ eluted with a $50 \mathrm{~mL}$ gradient from $0-500 \mathrm{mM} \mathrm{NaCl}$ in $25 \mathrm{mM}$ Tris, $\mathrm{pH} 7.4$ buffer. Pure ${ }_{403}$ 
proteins were dialyzed overnight against 2L of $25 \mathrm{mM}$ TES (or Tris), $100 \mathrm{mM} \mathrm{NaCl,} \mathrm{pH} 404$ 7.4, containing 2 g Chelex-100 resin (BioRad) to remove divalent metals. After the final 405 purification step, the purity of proteins products was assessed by SDS PAGE and MALDI- ${ }_{406}$ TOF mass spectrometry to be $>95$. Final protein products were flash frozen, dropwise, 407 in liquid nitrogen and stored at $-80^{\circ} \mathrm{C}$. Protein yields were typically on the order of 25408 $\mathrm{mg} / 1.5 \mathrm{~L}$ of culture.

\section{Preparation of biotinylated proteins for phage display}

A mutant version of hA5 with a single N-terminal Cys residues were generated via site- ${ }^{411}$ directed mutagenesis using the QuikChange lightning system (Agilent). The Cys was intro- ${ }_{412}$ duced in the Ser-Asn tag leftover from TEV protease cleavage as Ser-Asn-Cys. The proteins ${ }_{413}$ were expressed and purified as described in the previous section. A small amount of the ${ }_{414}$ purified proteins were biotinylated using the EZ-link BMCC-biotin system (ThermoFisher ${ }_{415}$ Scientific). $\approx 1 \mathrm{mg}$ BMCC-biotin was dissolved directly in 100\% DMSO to a concentration ${ }_{416}$ of $8 \mathrm{mM}$ for labeling. Proteins were exchanged into $25 \mathrm{mM}$ phosphate, $100 \mathrm{mM} \mathrm{NaCl}, \mathrm{pH}{ }_{417}$ 7.4 using a Nap-25 desalting column (GE Health Science) and degassed for $30 \mathrm{~min}$ at $25^{\circ} \mathrm{C}{ }_{418}$ using a vacuum pump (Malvern Instruments). While stirring at room temperature, $8 \mathrm{mM}{ }_{419}$ BMCC-biotin was added dropwise to a final 10X molar excess. Reaction tubes were sealed 420 with PARAFILM (Bemis) and the maleimide-thiol reactions were allowed to proceed for $1{ }_{421}$ hour at room temperature with stirring. The reactions were then transferred to $4^{\circ} \mathrm{C}$ and ${ }_{422}$ incubated with stirring overnight to allow completion of the reaction. Excess BMCC-biotin 423 was removed from the labeled proteins by exchanging again over a Nap-25 column (GE ${ }_{424}$ Health Science), and subsequently a series of 3 concentration-wash steps on a NanoSep 3K ${ }_{425}$ spin column (Pall corporation), into the Ca-TeBST loading loading buffer. Complete label- ${ }_{426}$ ing was confirmed by MALDI-TOF mass spectrometry by observing the $\approx 540 \mathrm{Da}$ shift in the ${ }_{427}$ protein peak. Final stocks of labeled proteins were prepared at $10 \mu M$ by dilution into the ${ }_{428}$ loading buffer. 


\section{Phage display}

Phage display experiments were performed using the $\mathrm{PhD}-12$ peptide phage display kit 431 (NEB). All steps involving the pipetting of phage-containing samples was done using fil- ${ }_{432}$ ter tips (Rainin). We prepared $100 \mu L$ samples containing phage $\left(5.5 \times 10^{11} \mathrm{PFU}\right)$ and ${ }_{433}$ $0.01 \mu M$ biotin-protein (or biotin alone in the negative control) at room temperature in a 434 background of $\mathrm{Ca}^{2+}$-TeBST loading buffer (50mM TES, $100 \mathrm{mM} \mathrm{NaCl,} 2 \mathrm{mM} \mathrm{CaCl}, 0.01 \% \quad 435$ Tween-20, $\mathrm{pH} 7.4)$ to ensure $\mathrm{Ca}^{2+}$-saturation of the S100 proteins. For the experiments 436 using a peptide competitor, we included the peptide RSHSGFDWRWAMEALTGGSAE at ${ }_{437}$ $20 \mu M$ in the loading buffer. This peptide (named A6cons in the original report), binds all ${ }_{438}$ four proteins at the canonical binding site with $K_{D}$ between 1 and $8 \mu M$ (Wheeler et al. , 439 2017). Samples were incubated at room temperature for $2 \mathrm{hr}$. Each sample was then applied 440 to one well of a 96-well high-capacity streptavidin plate (previously blocked using PhD-12 441 kit blocking buffer and washed 6X with $150 \mu L$ loading buffer). Samples were incubated 442 on the plate with gentle shaking for 20min. $1 \mu L$ of $10 m M$ biotin (NEB) was then added ${ }_{443}$ to each sample on the plate and incubated for an additional five minutes to compete away ${ }_{444}$ purely biotin-dependent interactions. Samples were then pulled from the plate carefully by 445 pipetting and discarded. Each well was washed $5 \mathrm{X}$ with $200 \mu L$ of loading buffer by applying ${ }_{446}$ the solution to the well and then immediately pulling off by pipetting. Finally, $100 \mu L$ of ${ }_{447}$ EDTA-TeBST elution buffer (50mM TES, 100mM NaCl, 5mM EDTA, 0.01\% Tween-20, pH ${ }_{448}$ 7.4) was applied to each well and the plate was incubated with gentle shaking for 1 hr at ${ }_{449}$ room temperature to elute. Eluates were pulled from the plate carefully by pipetting and 450 stored at $4^{\circ} \mathrm{C}$. Eluates were titered to quantify eluted phage as follows. Serial dilutions ${ }_{451}$ of the eluates from 1:10-1:105 were prepared in LB medium. These were used to 452 inoculate $200 \mu L$ aliquots of mid-log-phase ER2738 E. coli (NEB) by adding $10 \mu L$ to each. ${ }^{453}$ Each $200 \mu L$ aliquot was then mixed with $3 \mathrm{~mL}$ of pre-melted top agar, applied to a LB ${ }_{454}$ agar XGAL/IPTG (Rx Biosciences) plate, and allowed to cool. The plates were incubated ${ }_{455}$ overnight at $37^{\circ} \mathrm{C}$ to allow formation of plaques. The next morning, blue plaques were 456 counted and used to calculate $\mathrm{PFU} / \mathrm{mL}$ phage concentration. Enrichment was calculated as ${ }^{457}$ a ratio of experimental samples to the biotin-only negative control. 
To generate the input phage library, the commercially-produced library was first screened ${ }_{459}$ in duplicate against each of the four proteins as described above. Each of these lineages was 460 subsequently amplified in ER2738 E. coli (NEB) as follows. 20mL 1:100 dilutions of an ${ }_{461}$ ER2738 overnight culture were prepared. Each 20mL culture was inoculated with one entire 462 sample of remaining phage eluate. The cultures were incubated at $37^{\circ} \mathrm{C}$ with shaking for $4.5 \quad 463$ hours to allow phage growth. Bacteria were then removed by centrifugation and the top $80 \% \quad 464$ of the culture was removed carefully with a filtered serological pipette and transferred to a 465 fresh tube containing 1/6 volume of PEG/NaCl (20\% w/v PEG-8000, 2.5M NaCl). Samples 466 were incubated overnight at $4^{\circ} \mathrm{C}$ to precipitate phage. Precipitated phage were isolated by 467 centrifugation and subsequently purified by an additional PEG/ $\mathrm{NaCl}$ precipitation on ice 468 for $1 \mathrm{hr}$. These individually amplified pools were then resuspended in $200 \mu L$ each of sterile ${ }_{469}$ loading buffer and mixed together to form a pre-conditioned library in order to minimize the 470 impact of sampling on the subsequent panning experiment. The pool was diluted 1:1 with 471 $100 \%$ glycerol and stored at $-20^{\circ} \mathrm{C}$ for use in the final panning experiments.

\section{Preparation of deep sequencing libraries}

Phage genomic ssDNA was isolated from leftover amplified eluates from each round of pan- 474 ning using the M13 spin kit (Qiagen). Products were stored in low TE buffer. These ssDNA 475 were used as the template for 2 replicate PCRs with the Cs1 forward (5'-ACACTGACGA- 476 CATGGTTCTACAGTGGTACCTTTCTATTCTCACTCT-3') and PhD96seq-Cs2 reverse 477 (5'-TACGGTAGCAGAGACTTGGTCTCCCTCATAGTTAGCGTAACG-3') primers. Pro@rs ucts were isolated from these PCR products using the GeneJet gel extraction kit (Thermo 479 Scientific) and pooled. The pooled products were then used as templates for a secondary 480 reaction with the barcoded primers. Products were isolated from these final PCRs using the ${ }_{481}$ GeneJet gel extraction kit. Concentration of barcoded samples was measured by $A_{260} / A_{280} 482$ using a 1mm cuvette on an Eppendorf biospectrometer. Multiplexing was done by mixing 483 samples according to mass. The concentration of the multiplexed library was corrected using 484 qPCR with the P5 and P7 Illumina flow-cell primers. The library was then diluted to a final 485 concentration of 10nM and Illumina sequenced on two lanes of a HiSeq 4000 instrument, 486 using the Cs1 F' as the R1 sequencing primer. The lanes were spiked with $20 \%$ PhiX control ${ }^{487}$ 


\section{Phage display analysis pipeline}

We performed quality control on three read features. First, we verified that the sequence 490 had exactly the anticipated length from the start of the phage sequence through the stop 491 codon. Second, we only took sequences in which the invariant phage sequence differed by 492 at most one base from the anticipated sequence. This allows for a single point mutation 493 and or sequencing errors, but not wholesale changes in the sequence. Finally, we took only 494 reads with an average phred score better than 15 . The vast majority of the reads that 495 failed our quality control did not have the variable region, representing reversion to phage 496 with a wildtype-like coat protein. This analysis is encoded in the hops_count.py script 497 (https://github.com/harmslab/hops), which takes a gzipped fastq file as input and returns 498 the counts for every peptide in the file.

\section{Identifying the read count cutoff}

One critical question is at what point the number of reads correlates with the frequency of 501 a peptide. If we set the cutoff too low, we incorporate noise into downstream analyses. If ${ }_{502}$ we set the cutoff too high, we remove valuable observations from our dataset. To identify 503 an appropriate cutoff, we studied the mapping between $c_{i}$ (the number of reads arising from 504 peptide $i$ ) and $f_{i}$ (the actual frequency of peptide $i$ in the experiments). Our goal was to 505 find $P\left(f_{i} \mid c_{i}, N\right)$ : the probability peptide $i$ is at $f_{i}$ given we observe it $c_{i}$ times in $N$ counts. 506 Using Bayes theorem, we can write

$$
P\left(f_{i} \mid c_{i}, N\right)=\frac{P\left(c_{i} \mid f_{i}, N\right) P\left(f_{i}\right)}{P\left(c_{i}\right)}
$$

where $N$ is the total number of reads. We calculated $P\left(c_{i} \mid f_{i}, N\right)$ assuming a binomial sam- 508 pling process: what is the probability of observing exactly $c$ counts given $N$ independent 509 samples when a population with a peptide frequency $f_{i}$ ? This gives the curve seen in Fig 510 $\mathrm{S} 2 \mathrm{~A}$. We then estimated $P\left(f_{i}\right)$ from the distribution of frequencies in the input library, con- 511 structing a histogram of apparent peptide frequencies (Fig S2B). Empirically, we found that 512 
frequencies followed an exponential distribution over the measurable range of frequencies. 513 Finally, we assumed that all counts have equal prior probabilities, turning $P\left(c_{i}\right)$ into a scalar 514 that normalizes the integral of $P\left(f_{i} \mid c_{i}, N\right)$ so it sums to $1 . \quad 515$

Using the information from Fig S2A and B, we could then calculate $P\left(f_{i} \mid c_{i}, N\right)$ for any 516 number of reads in an experiment $N$. Fig S2C shows this calculation for $N=2.0 \times 10^{7}{ }_{517}$ reads - a typical number of reads from our experimental replicates. This curve is linear above 518 6 reads. Below this, counts no longer correlates linearly with frequency, as it is possible to 519 obtain 5 reads random sampling from low frequency library members. We therefore used a 520 cutoff of 6 counts for all downstream analyses. In total, $74.0 \%$ of reads passed our quality 521 control and read cutoff (Table S1).

\section{Measuring enrichment values}

We next set out to measure changes in the frequency of peptides between the competitor ${ }_{524}$ and non-competitor samples. The simplest way to do this would be to identify peptides seen 525 in both experiments, and then measure how their frequencies change between conditions. 526 Unfortunately, these proteins all bind a wide swath of peptide targets and relatively few ${ }_{527}$ peptides were shared between conditions. This approach would thus exclude the majority 528 of sequences. For example, only 8,672 of the 112,681 unique peptides observed for hA5 were ${ }_{529}$ present in both the competitor and non-competitor, even after pooling biological replicates. 530 Worse, because we are interested in peptides that are lost when competitor peptide is added, 531 ignoring peptides with no counts in the competitor sample means ignoring some of the most 532 informative peptides.

To solve this problem, we clustered similar peptides and measured enrichment for peptide $\quad 534$ clusters rather than individual peptides. We extracted all peptides that were observed across 535 the competitor and non-competitor samples for a given protein. We then used DBSCAN to 536 cluster those peptides according to sequence similarity, as measured by their their Damerau- 537 Levenshtein distance (Damerau, 1964; Ester et al. , 1996). This revealed extensive structure 538 in our data. For example, hA5 yielded 8,645 clusters with more than one peptide, incor- 539 porating more than half of the unique peptides (Fig S3A). We chose clustering parameters 540 that led to highly similar peptides within each cluster, as can be seen by the representative ${ }_{541}$ 
sequence logos for three clusters of hA5 (Fig S3B). Sequences that were not placed in clusters 542 were treated as clusters with a size of one.

We then used the enrichment of each cluster to estimate the enrichment of individual 544 peptides. We defined enrichment as:

$$
E_{\text {cluster }}=-\ln \left(\frac{\sum_{i=1}^{i \leq N} \beta_{i}}{\sum_{i=1}^{i \leq N} \alpha_{i}}\right)
$$

where $N$ is the total number of peptides in the cluster, $\beta_{i}$ is the frequency of peptide $i$ in the ${ }_{546}$ competitor sample, and $\alpha_{i}$ is the frequency of peptide $i$ in the non-competitor sample. We ${ }_{547}$ then made the approximation that all members of the cluster have the same enrichment: ${ }_{548}$

$$
E_{i} \approx E_{\text {cluster }}
$$

allowing us to estimate the enrichment of all $i$ peptides in the cluster (Fig S3C). Peptides 549 lost because of competition for the interface will add zeros to the numerator of Eq. 1, leading 550 to an overall decrease in enrichment. Peptides missed because of finite sampling will add 551 zeros evenly to the competitor and non-competitor samples, leading to no net enrichment. ${ }_{552}$

We tested this cluster-based approximation using the 8,672 peptides of hA5 for which we 553 could directly calculate enrichment (that is, those peptides seen in both the competitor and 554 non-competitor experiments). We calculated the enrichment of each peptide individually 555 and compared these values to those obtained by the cluster method. There is no systematic 556 difference in the values estimated using the two methods, and the linear model explains 557 $98.4 \%$ of the variation between the two methods.

We clustered peptides using our own implementation of the DBSCAN algorithm (Es- 559 ter et al. , 1996) using the Damerau-Levensthein distance (Damerau, 1964). The main 560 parameter for DBSCAN clustering is $\varepsilon$ - the neighborhood cutoff. Clusters are defined as 561 sequences that can be reached through a series of $\varepsilon$-step moves. We found that $\varepsilon=1562$ gave the best results for our downstream machine learning analysis. Our whole enrichment 563 pipeline - including clustering - can be run given a peptide count file for the non-competitor 564 experiment and a peptide-count file for the competitor experiment using the hops_enrich.py 565 


\section{Acknowledgements}

We'd like to thank members of the Harms lab for critical feedback on the manuscript. This 568 work was supported by NIH R01GM117140 (MJH) and NIH 7T32GM007759-37 (LCW). ${ }_{569}$

\section{References}

Alhindi, T., Zhang, Z., Ruelens, P., Coenen, H., Degroote, H., Iraci, N., \& Geuten, K. 2017.571 Protein interaction evolution from promiscuity to specificity with reduced flexibility in an 572 increasingly complex network. Scientific Reports, 7 (Mar.).

573

Bertini, Ivano, Gupta, Soumyasri Das, Hu, Xiaoyu, Karavelas, Tilemachos, Luchinat, Clau- 574 dio, Parigi, Giacomo, \& Yuan, Jing. 2009. Solution Structure and Dynamics of S100A5 in 575 the Apo and Ca2+-Bound States. JBIC Journal of Biological Inorganic Chemistry, 14(7), 576 $1097-1107$.

Carroll, Sean Michael, Bridgham, Jamie T., \& Thornton, Joseph W. 2008. Evolution of Hor- 578 mone Signaling in Elasmobranchs by Exploitation of Promiscuous Receptors. Molecular 579 Biology and Evolution, 25(12), 2643-2652.

Clifton, Ben E., \& Jackson, Colin J. 2016. Ancestral Protein Reconstruction Yields Insights 581 into Adaptive Evolution of Binding Specificity in Solute-Binding Proteins. Cell Chemical ${ }_{582}$ Biology, 23(2), 236-245.

Copley, Shelley D. 2012. Toward a Systems Biology Perspective on Enzyme Evolution. The 584 Journal of Biological Chemistry, 287(1), 3-10.

Damerau, Fred J. 1964. A Technique for Computer Detection and Correction of Spelling 586 Errors. Commun. ACM, 7(3), 171-176.

Devamani, Titu, Rauwerdink, Alissa M., Lun-zer, Mark, Jones, Bryan J., Mooney, Joanna L., 588 Tan, Maxilmilien Alaric O., Zhang, Zhi-Jun, Xu, Jian-He, Dean, Antony M., \& Kazlauskas, ${ }^{589}$ 
Romas J. 2016. Catalytic promiscuity of ancestral esterases and hydroxynitrile lyases. 590 Journal of the American Chemical Society, 138(3), 1046-1056.

Donato, R., R Cannon, B., Sorci, G., Riuzzi, F., Hsu, K., J Weber, D., \& L Geczy, C. 2013.592 Functions of S100 Proteins. Current molecular medicine, 13(1), 24-57.

Eick, Geeta N., Colucci, Jennifer K., Harms, Michael J., Ortlund, Eric A., \& Thornton, 594 Joseph W. 2012. Evolution of Minimal Specificity and Promiscuity in Steroid Hormone 595 Receptors. PLoS Genetics, 8(11).

Eick, Geeta N., Bridgham, Jamie T., Anderson, Douglas P., Harms, Michael J., \& Thornton, 597 Joseph W. 2017. Robustness of Reconstructed Ancestral Protein Functions to Statistical 598 Uncertainty. Molecular Biology and Evolution, 34(2), 247-261.

Ester, Martin, Kriegel, Hans-Peter, Sander, Jörg, \& Xu, Xiaowei. 1996. A Density-Based 600 Algorithm for Discovering Clusters in Large Spatial Databases with Noise. AAAI Press. ${ }_{601}$

Gaucher, Eric A., Govindarajan, Sridhar, \& Ganesh, Omjoy K. 2008. Palaeotemperature 602 trend for Precambrian life inferred from resurrected proteins. Nature, 451(7179), 704-707. 603

Hittinger, Chris Todd, \& Carroll, Sean B. 2007. Gene duplication and the adaptive evolution 604 of a classic genetic switch. Nature, 449(7163), 677-681.

Jensen, R. A. 1976. Enzyme Recruitment in Evolution of New Function. Annual Review of ${ }_{606}$ Microbiology, 30(1), 409-425.

Kaltenbach, Miriam, \& Tokuriki, Nobuhiko. 2014. Dynamics and constraints of enzyme evo- ${ }^{6} 08$ lution. Journal of Experimental Zoology Part B: Molecular and Developmental Evolution, 609 $322(7), 468-487$.

Kanzaki, Hiroyuki, Yoshida, Kentaro, Saitoh, Hiromasa, Fujisaki, Koki, Hirabuchi, Akiko, 611 Alaux, Ludovic, Fournier, Elisabeth, Tharreau, Didier, \& Terauchi, Ryohei. 2012. Arms 612 race co-evolution of Magnaporthe oryzae AVR-Pik and rice Pik genes driven by their ${ }_{613}$ physical interactions. The Plant Journal, 72(6), 894-907. 
Khersonsky, Olga, \& Tawfik, Dan S. 2010. Enzyme Promiscuity: A Mechanistic and Evolu- 615 tionary Perspective. Annual Review of Biochemistry, 79(1), 471-505.

616

Leclerc, Estelle, Fritz, Günter, Vetter, Stefan W., \& Heizmann, Claus W. 2009. Binding of 617 S100 Proteins to RAGE: An Update. Biochimica et Biophysica Acta (BBA) - Molecular 618 Cell Research, 1793(6), 993-1007.

Lee, Young-Tae, Dimitrova, Yoana N., Schneider, Gabriela, Ridenour, Whitney B., Bhat- ${ }_{620}$ tacharya, Shibani, Soss, Sarah E., Caprioli, Richard M., Filipek, Anna, \& Chazin, Wal- ${ }^{621}$ ter J. 2008. Structure of the S100A6 Complex with a Fragment from the C-Terminal 622 Domain of Siah-1 Interacting Protein: A Novel Mode for S100 Protein Target Recogni- ${ }^{623}$ tion. Biochemistry, 47(41), 10921-10932.

Liriano, Melissa A. 2012. Structure, Dynamics and Function of S100B and S100A5 Com- 625 plexes. Ph.D., University of Maryland, Baltimore, United States - Maryland.

Ma, Sheng, Martin-Laffon, Jacqueline, Mininno, Morgane, Gigarel, Océane, Brugière, 627 Sabine, Bastien, Olivier, Tardif, Marianne, Ravanel, Stéphane, \& Alban, Claude. 2016. ${ }^{628}$ Molecular Evolution of the Substrate Specificity of Chloroplastic Aldolases/Rubisco Ly- ${ }_{629}$ sine Methyltransferases in Plants. Molecular Plant, 9(4), 569-581.

Mannige, Ranjan V., Brooks, Charles L., \& Shakhnovich, Eugene I. 2012. A Universal 631 Trend among Proteomes Indicates an Oily Last Common Ancestor. PLOS Computational 632 Biology, 8(12), e1002839.

Pougach, Ksenia, Voet, Arnout, Kondrashov, Fyodor A., Voordeckers, Karin, Christiaens, 634 Joaquin F., Baying, Bianka, Benes, Vladimir, Sakai, Ryo, Aerts, Jan, Zhu, Bo, Van Dijck, ${ }_{635}$ Patrick, \& Verstrepen, Kevin J. 2014. Duplication of a promiscuous transcription factor ${ }_{636}$ drives the emergence of a new regulatory network. Nature Communications, 5(Sept.). 637

Rauwerdink, Alissa, Lunzer, Mark, Devamani, Titu, Jones, Bryan, Mooney, Joanna, Zhang, 638 Zhi-Jun, Xu, Jian-He, Kazlauskas, Romas J., \& Dean, Antony M. 2016. Evolution of a 639 Catalytic Mechanism. Molecular Biology and Evolution, 33(4), 971-979. 
Reinke, Aaron W., Baek, Jiyeon, Ashenberg, Orr, \& Keating, Amy E. 2013. Networks of 641 bZIP Protein-Protein Interactions Diversified Over a Billion Years of Evolution. Science, 642 340(6133), 730-734.

Risso, Valeria A., Gavira, Jose A., Mejia-Carmona, Diego F., Gaucher, Eric A., \& Sanchez- ${ }^{644}$ Ruiz, Jose M. 2013. Hyperstability and Substrate Promiscuity in Laboratory Resurrections 645 of Precambrian beta-Lactamases. Journal of the American Chemical Society, 135(8), 646 2899-2902.

Risso, Valeria A., Gavira, Jose A., \& Sanchez-Ruiz, Jose M. 2014. Thermostable and promis- ${ }_{648}$ cuous Precambrian proteins. Environmental Microbiology, 16(6), 1485-1489.

Risso, Valeria A, Sanchez-Ruiz, Jose M, \& Ozkan, S Banu. 2018. Biotechnological and 650 protein-engineering implications of ancestral protein resurrection. Current Opinion in 651 Structural Biology, 51, 106 - 115. Engineering and design: New applications • Membranes. 652

Santamaria-Kisiel, Liliana, Rintala-Dempsey, Anne C., \& Shaw, Gary S. 2006. Calcium- ${ }^{653}$ dependent and -independent interactions of the S100 protein family. Biochemical Journal, ${ }_{654}$ 396(2), 201-214.

Sidhu, Sachdev S., Lowman, Henry B., Cunningham, Brian C., \& Wells, James A. 2000. 656 Phage Display for Selection of Novel Binding Peptides. Methods in Enzymology, 328(Jan.), 657 333-IN5.

$\mathrm{S} \backslash$ lomnicki, \Lukasz P., Nawrot, Barbara, \& Leśniak, Wies \lawa. 2009. S100A6 Binds P53 659 and Affects Its Activity. The International Journal of Biochemistry \& Cell Biology, 41(4), 660 784-790.

Soskine, Misha, \& Tawfik, Dan S. 2010. Mutational effects and the evolution of new protein ${ }_{662}$ functions. Nature Reviews Genetics, 11(8), 572-582.

Streicher, Werner W., Lopez, Maria M., \& Makhatadze, George I. 2009. Annexin I and 664 Annexin II N-Terminal Peptides Binding to S100 Protein Family Members: Specificity 665 and Thermodynamic Characterization. Biochemistry, 48(12), 2788-2798. 
Taverna, Darin M., \& Goldstein, Richard A. 2002. Why are proteins marginally stable? ${ }_{667}$ Proteins: Structure, Function, and Bioinformatics, 46(1), 105-109.

Wheeler, Lucas C, Lim, Shion A, Marqusee, Susan, \& Harms, Michael J. 2016. The ther- ${ }_{669}$ mostability and specificity of ancient proteins. Current Opinion in Structural Biology, 670 38(June), 37-43.

Wheeler, Lucas C., Anderson, Jeremy A., Morrison, Annelise J., Wong, Caitlyn E., \& Harms, 672 Michael J. 2017. Conservation of specificity in two low-specificity proteins. bioRxiv, Oct., 673 207324.

Willats, William G. T. 2002. Phage Display: Practicalities and Prospects. Plant Molecular 675 Biology, 50(6), 837-854.

Williams, Paul D., Pollock, David D., Blackburne, Benjamin P., \& Goldstein, Richard A. 677 2006. Assessing the Accuracy of Ancestral Protein Reconstruction Methods. PLOS Com- 678 putational Biology, 2(6), e69.

Zou, Taisong, Risso, Valeria A., Gavira, Jose A., Sanchez-Ruiz, Jose M., \& Ozkan, S. Banu. ${ }^{680}$ 2015. Evolution of Conformational Dynamics Determines the Conversion of a Promiscuous ${ }_{681}$ Generalist into a Specialist Enzyme. Molecular Biology and Evolution, 32(1), 132-143. 682 


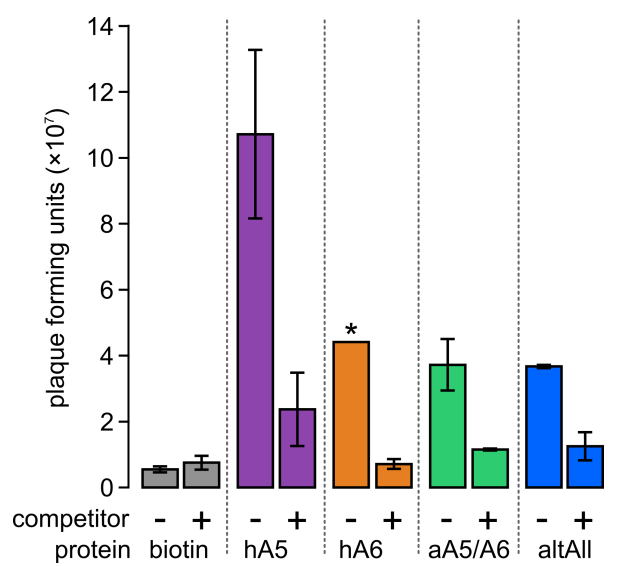

Fig S1. Phage enrichment is reduced in the presence of competitor peptide. 684 Figure shows eluted plaque forming units (PFU) (estimated from phage titer) for two bi- 685 ological replicates of each condition. Enrichment is shown for biotin-only control (gray), 686 hA5 (purple), hA6 (orange), ancA5/A6 (dark green), and altAll (light green) with $(+)$ and 687 without (-) $20 \mu M$ competitor peptide. Error bars show the standard error for two biological 688 replicates. $\left.*^{*}\right)$ hA6 without competitor is shown for only one replicate due to failure of the 689 titer for the one replicate. 

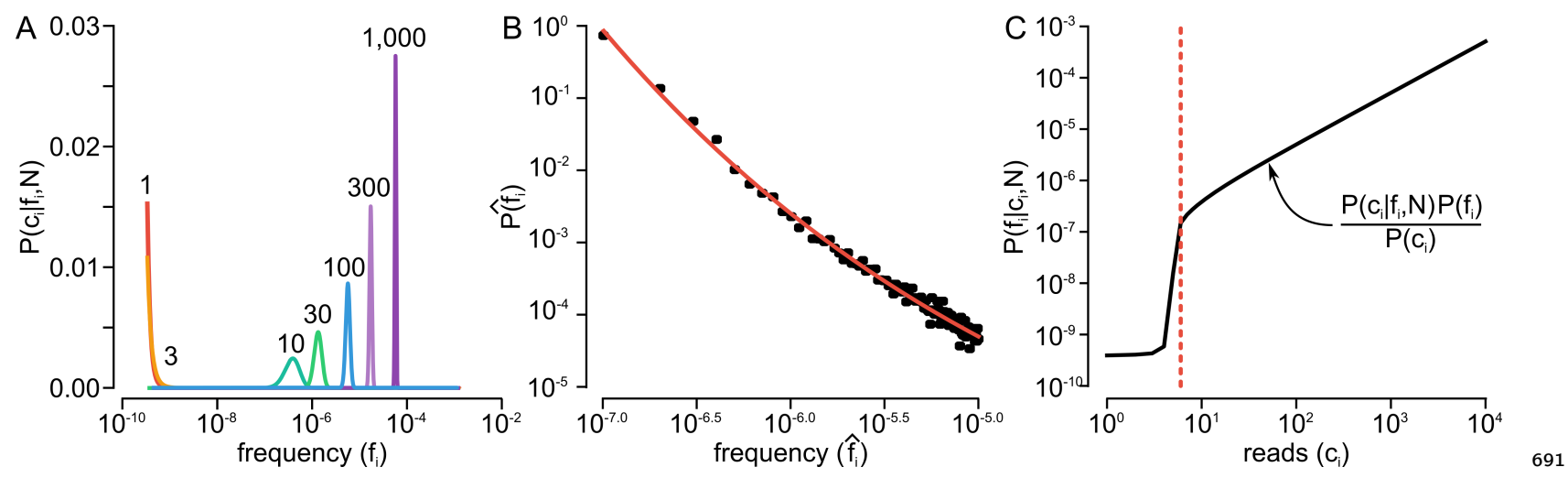

Fig S2. We can identify the number of counts that reliably reports on frequency 692 in a sequenced phage pool. A) Using binomial sampling, we can calculate the probability 693 of observing exactly $c_{i}$ counts in $N$ samples from a pool that has a peptide of actual frequency 694 $f_{i}$. Figure shows curves for counts ranging from 1 (red) to 1,000 (pink), all using $N=695$ $2.0 \times 10^{7}$. B) Panel shows a histogram of frequencies estimated from $3.9 \times 10^{7}$ reads taken from 696 the input library. The black points are experimental data. The red curve is an exponential 697 distribution fit to that curve. C) Using the sampling from panel A and the fit curve from 698 panel B, we can determine $P\left(f_{i} \mid c_{i}, N\right)$. The solid curve shows the relationship between ${ }^{699}$ the number of reads for peptide $i$ (x-axis) against the maximum-likelihood estimate of the 700 frequency (y-axis). The red line highlights the cutoff we used in our experiments. 701 

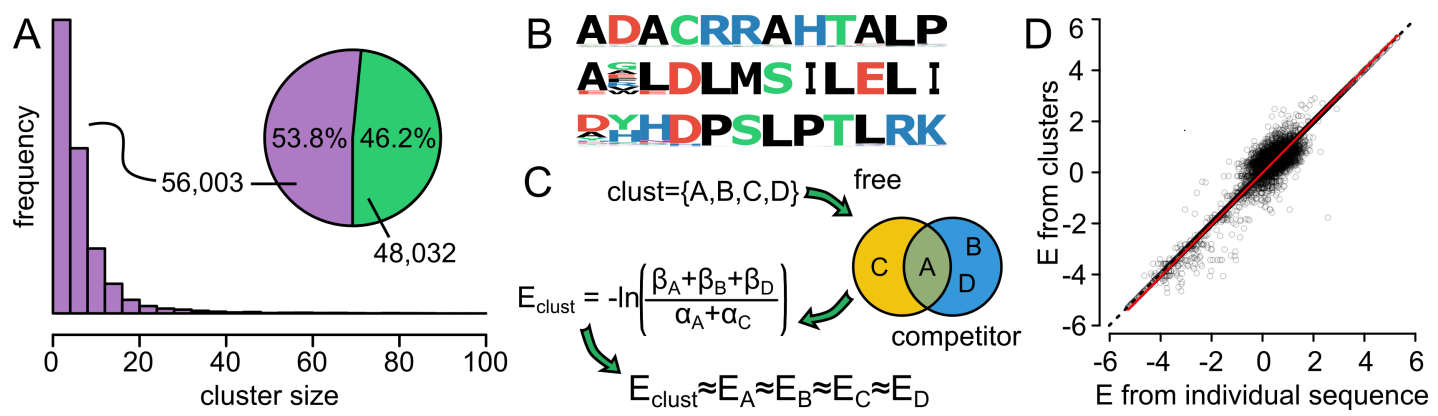

702

Fig S3. We can estimate how addition of competitor peptide alters the frequen- 703 cies of peptides. A) Distribution of sizes of peptide clusters from hA5 experiment. Pie 704 chart shows number of peptides placed in clusters $(56,003 ; 53.8 \%)$ versus not $(48,032 ; 46.2 \%)$. 705 B) Three example clusters taken from the clusters in panel A. The letter height at each po- 706 sition indicates its frequency in the sequences within that cluster. C) Toy example showing 707 how enrichment is calculated for a cluster containing peptides $\{A, B, C, D\}$. Peptides $A$ and 708 $C$ were observed in the no competitor sample at frequencies $\alpha_{A}$ and $\alpha_{C}$. Peptides $A, B$, and 709 $D$ were observed in the competitor sample at frequencies $\beta_{A}, \beta_{B}$ and $\beta_{D}$. The enrichment 710 of the cluster is given by $E_{\text {clust }}=-\ln \left[\left(\beta_{A}+\beta_{B}+\beta_{D}\right) /\left(\alpha_{A}+\alpha_{C}\right)\right]$. All members of the 711 cluster are then assigned $E \approx E_{\text {clust }}$. D) Comparison of enrichment values for hA5 peptides 712 determined using a direct comparison of frequencies with and without competitor (x-axis) 713 versus the clustering method (y-axis). Each point is an individual peptide. Red line is a 714 least-squares regression line fit to the data. The dashed line is the 1:1 line. 

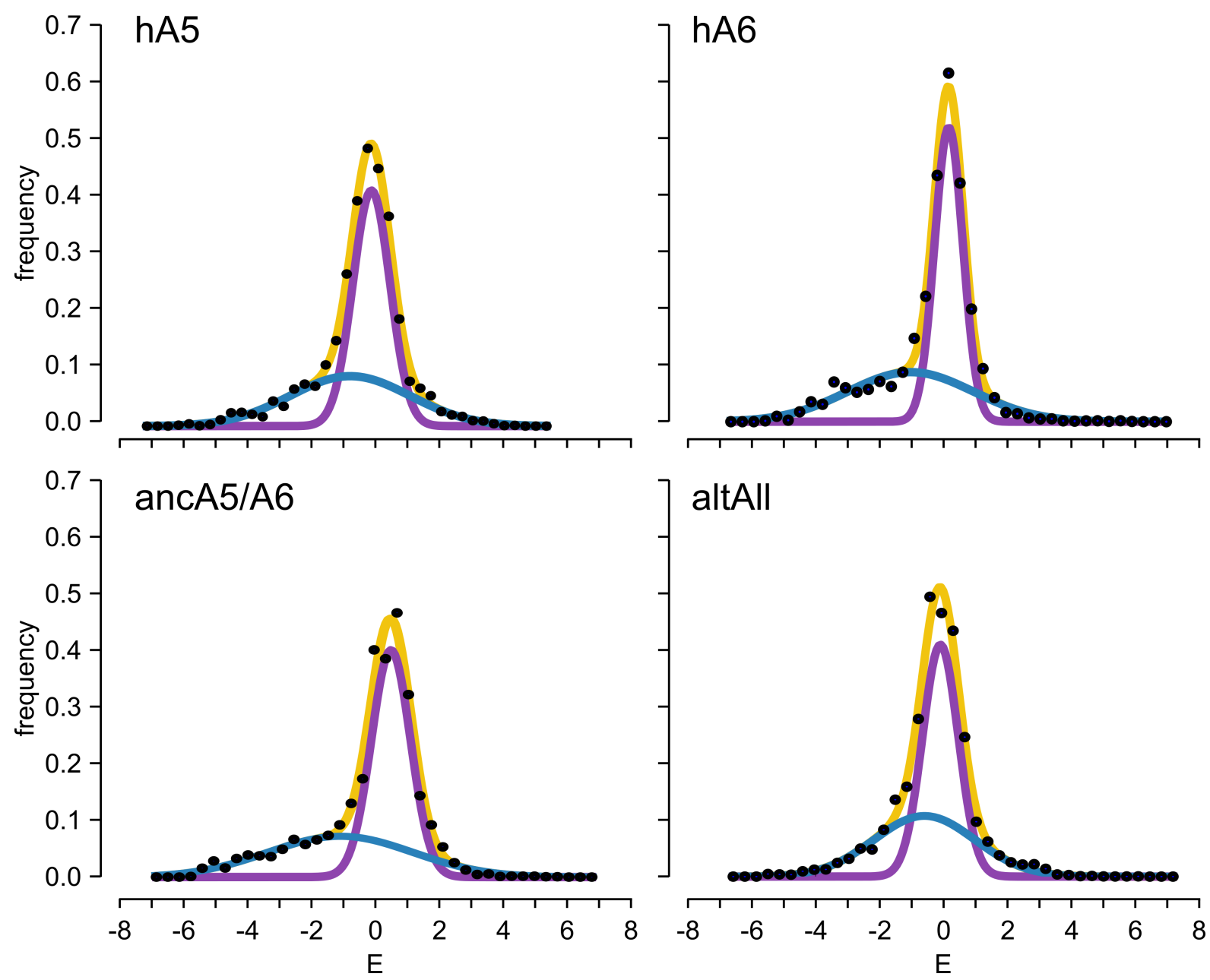

Fig S4. Distributions of $E$ for each protein. Panels show distribution of $E$ for each 717 protein (pooled bio-replicates). Points are raw histograms. Curves are two Gaussian fit: 718 blue (responsive), purple (unresponsive) and yellow (sum). 


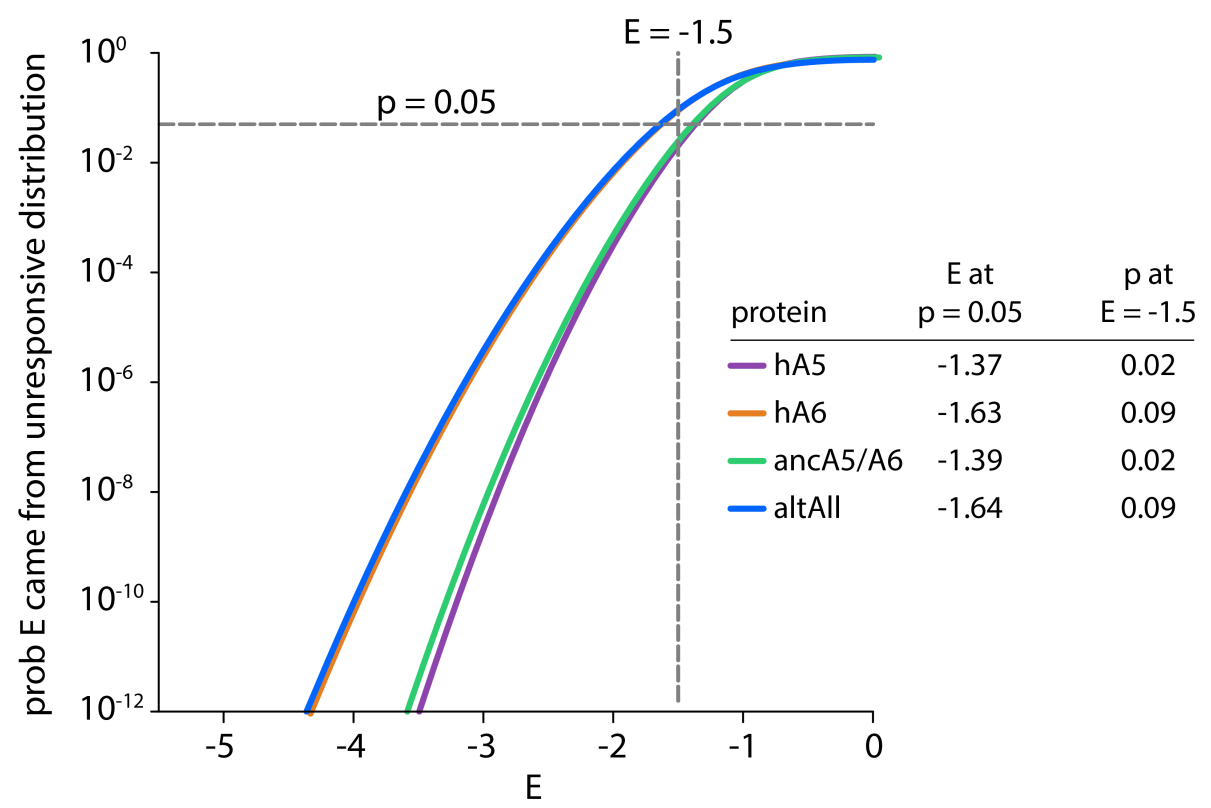

Fig S5. Selection of $E=-1.5$ as a cutoff for peptide enrichment. Curves show the 722 prosterior probability a peptide with the enrichment score shown on the x-axis arose from 723 the "unresponsive" distribution for each protein. These curves were calculated by

$$
P_{\text {unresponsive }}(E)=\frac{p d f_{\text {unresponsive }}(E)}{p d f_{\text {responsive }}(E)+p d f_{\text {unresponsive }}(E)}
$$

where $p d f$ is the probability density function in $E$ for the normal distributions seen in Fig 725 $2 \mathrm{C}$ and S4. The inset table shows the $E$ value where the $P_{\text {unresponsive }}(E)$ is 0.05 and the 726 $\begin{array}{ll}P_{\text {unresponsive }}(E) \text { at } E=-1.5 . & 727\end{array}$ 
Table S1: Number of sequencing reads for each sample. Sample, and whether or 729 not competitor was added, are indicated on the right. Columns show biological replicates 1730 or 2. "total" columns indicate reads returned by Illumina software pipeline. "good" columns 731 indicate reads that passed our quality control and were used to calculate enrichment values. 732

\begin{tabular}{cc|rr|rr} 
& & & \multicolumn{2}{|c|}{ rep1 } & \multicolumn{2}{c}{ rep2 } \\
sample & competitor & total & good & total & good \\
\hline hA5 & - & $24,794,016$ & $19,695,958$ & $29,085,203$ & $16,773,567$ \\
hA5 & + & $15,053,706$ & $11,523,991$ & $17,631,137$ & $13,612,463$ \\
\hline hA6 & - & $22,728,393$ & $17,722,779$ & $7,769,003$ & $5,972,295$ \\
hA6 & + & $13,953,466$ & $11,004,701$ & $23,026,469$ & $18,128,759$ \\
\hline raw library & & $39,700,991$ & $32,190,368$ & &
\end{tabular}

